\title{
FETAL MODEL OF SINGLE VENTRICLE PHYSIOLOGY: HEMODYNAMIC EFFECTS OF OXYGEN, NITRIC OXIDE, CARBON DIOXIDE, AND HYPOXIA IN THE EARLY POSTNATAL PERIOD
}

V. Mohan Reddy, MD

John R. Liddicoat, MD

Jeffrey R. Fineman, MD

Doff B. McElhinney, MS

Judith R. Klein, MD

Frank L. Hanley, MD
Background: In patients with single ventricle physiology, the distribution of flow to the systemic and pulmonary circulations, which are in parallel, largely depends on the relative resistances in the respective vascular beds. Although neonatal palliation in patients with single ventricle physiology has become more common, medical management during the perinatal and perioperative periods is often based primarily on personal and institutional experience and is complicated by the transition to postnatal life and the effects of cardiopulmonary bypass. The lack of an animal model that suitably mimics single ventricle physiology has impeded progress in this area. Objective: The purpose of the current study was to investigate the effects of respiratory manipulations on pulmonary hemodynamics in the early neonatal period in a reproducible model of single ventricle physiology created in utero. Methods: A $10 \mathrm{~mm}$ Damus-Kaye-Stansel aortopulmonary anastomosis was created in fetal sheep ( $n=14$ ) at $140 \pm 1.2$ days' gestation, with pulmonary blood flow provided through a $5 \mathrm{~mm}$ aortopulmonary shunt after ligation of the main pulmonary artery distally. Two to 3 days after delivery at term, lambs $(n=11)$ underwent an open sternotomy and 30 minutes of deep hypothermic circulatory arrest. Both before and after bypass, respiratory manipulations, including administration of nitric oxide (80 ppm), 100\% oxygen, $10 \%$ oxygen, and 5\% carbon dioxide, were performed and hemodynamic variables were measured. Results: Nitric oxide and oxygen caused a decrease in pulmonary vascular resistance and an increase in the pulmonary/systemic blood flow ratio, both before and after bypass. Hypoxia and carbon dioxide produced a significant rise in pulmonary vascular resistance and a significant drop in the ratio of pulmonary to systemic blood flow. Conclusions: Oxygen, nitric oxide, and carbon dioxide all appear to be useful means of manipulating pulmonary vascular resistance and pulmonary/systemic blood flow ratio in neonatal lambs with single ventricle physiology, but further investigation is necessary to understand their dose responsive effects and the effects of prolonged administration. (J Thorac Cardiovasc Surg 1996;112:437-49)
P atients born with congenital heart lesions characterized by one functioning ventricle, and hence parallel systemic and pulmonary circulations, often

From the Division of Cardiothoracic Surgery, University of California San Francisco, San Francisco, Calif.

Presented in part at the Sixty-seventh Annual Scientific Session of the American Heart Association, Dallas, Tex., November 13-17, 1994.

Supported in part by funding from the National Institutes of Health: 2 RO1 HL43357.

Received for publication Sept. 21, 1995; revisions requested Nov. 29, 1995; revisions received Feb. 9, 1996; accepted for publication Feb. 13, 1996.

Address for reprints: V. Mohan Reddy, MD, 505 Parnassus Ave., M593, San Francisco, CA 94143-0118.

Copyright (C) 1996 by Mosby-Year Book, Inc.

$0022-5223 / 96 \$ 5.00+0 \quad \mathbf{1 2 / 1 / 7 2 6 6 0}$ depend for their pulmonary blood flow on systemicpulmonary arterial communications, via either a patent ductus arteriosus or a surgically created shunt. In this setting, pulmonary and systemic blood flows ( $Q p$ and $Q s$, respectively) are a function of relative resistances in the respective vascular beds and are among the primary determinants of systemic arterial oxygen saturation. ${ }^{1}$ An important goal in the preoperative and postoperative management of these patients is to minimize the total volume work of the single ventricle and preserve adequate systemic oxygen delivery by achieving a critical balance between Qp and Os. Various ventilatory adjustments, inhalational agents, and pharmacologic agents are commonly used to achieve such a balance. $^{1,2}$ Although the effects of inspired gases, $\mathrm{pH}$, 


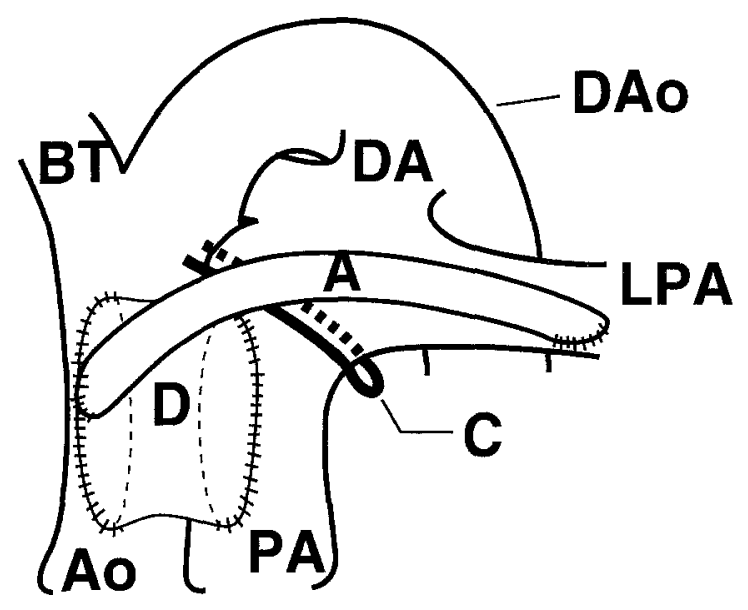

Fig. 1. Completed surgical procedure for in utero creation of Damus-Kaye-Stansel anastomosis to achieve single ventricle physiology. $A$, Aortopulmonary shunt; $A o$, ascending aorta; $B T$, brachiocephalic trunk; $C$, clip ligation of main pulmonary artery; $D$, Damus-Kaye-Stansel anastomosis; $D A$, ductus arteriosus; $D A o$, descending aorta; $L P A$, left pulmonary artery; $P A$, main pulmonary artery.

and other pharmacologic agents on pulmonary vasculature are well understood in the in-series twoventricle circulation, ${ }^{3-9}$ little such information is available in the setting of single ventricle physiology. Therefore controversies exist in the management of patients with single ventricle physiology, because often the decisions are based on individual institutional experiences ${ }^{10-12}$ derived from monitoring physiologic variables that only indirectly reflect the changes in the systemic (SVR) and pulmonary (PVR) vascular resistances. Recently reported experimental $^{13}$ and mathematical ${ }^{14}$ models of single ventricle physiology have provided some insight into the management of these patients. However, these models do not adequately account for the transitional physiology, either because they were created postnatally $^{13}$ or because they are purely theoretical. ${ }^{14}$ We have recently established a reliable fetal animal model of single ventricle physiology. In the current report we examine the effects of inhalational agents, including nitric oxide, $100 \%$ oxygen, $10 \%$ oxygen, and $5 \%$ carbon dioxide, on pulmonary and systemic hemodynamics in this model.

\section{Materials and methods}

Surgical preparation and care

Ewes and fetuses. Fourteen mixed-breed Western ewes $(140 \pm 1.2$ days' gestation, term $=145$ to 150 days $)$ were operated on under sterile conditions with the use of local anesthesia ( $2 \%$ lidocaine hydrochloride), epidural anesthesia ( $4 \mathrm{ml}$ of $1 \%$ tetracaine hydrochloride), and intravenous sedation ( 50 to $100 \mathrm{mg}$ ketamine hydrochloride). After placement of a central venous catheter into the maternal jugular vein, a midline laparotomy was performed, and the horn of the uterus containing the fetus was exposed. Hysterotomy was performed, the left fetal forelimb was extracted, and fetal anesthesia was administered with ketamine hydrochloride $(10 \mathrm{mg} / \mathrm{kg}$ intramuscularly) and succinylcholine hydrochloride $(2 \mathrm{mg} / \mathrm{kg}$ intramuscularly) to prevent fetal respiratory movements. The chest of the fetus was exposed and 1\% lidocaine was injected along the line of incision. A left lateral thoracotomy was performed in the third intercostal space, after which an oblique pericardiotomy was made and the great vessels were exposed. The ascending aorta, main pulmonary artery, and brachiocephalic arterial trunk were dissected and controlled with vessel loops. The Damus-KayeStansel anastomosis was performed in the following manner (Fig. 1). A side-biting vascular clamp was placed on the ascending aorta and an aortotomy was performed with a No. 11 blade knife. The aortotomy was extended to about $10 \mathrm{~mm}$ in length with fine scissors, and a strip of aortic wall was excised to create an oval opening in the ascending aorta. A short piece (about $10 \mathrm{~mm}$ length) of 10 mm polytetrafluoroethylene vascular graft* was anastomosed end to side to the ascending aorta with 7-0 Prolene sutures (Ethicon, Inc., Somerville, N.J.) with a continuous suture technique. The vascular clamp was then applied to the main pulmonary artery. A pulmonary arteriotomy was performed and a strip of the posterior pulmonary arterial wall was excised. The free end of the $10 \mathrm{~mm}$ graft was anastomosed end to side to the pulmonary artery. The vascular clamp was gradually released, allowing any air in the graft to escape through the suture line and needle holes, and the vascular clip was removed to establish graft patency. The main pulmonary artery was occluded distal to the Damus anastomosis with a large vascular clip. An aortopulmonary shunt was then created with a $5 \mathrm{~mm}$ polytetrafluoroethylene tube graft from the ascending aorta to the left pulmonary artery (Fig. 1). The thoracotomy incision was closed in layers. Warm saline solution was infused to replace the lost amniotic fluid and the uterine incision was closed. After recovery from anesthesia, the ewe was returned to the cage with free access to food and water. Antibiotics ( 2 million units of penicillin $G$ procaine and $100 \mathrm{mg}$ of gentamicin sulfate) were administered intravenously to the ewe during the operation and daily thereafter for 2 more days.

Lambs. After spontaneous delivery, the lambs were allowed to have a normal postnatal course for 48 to 72 hours, after which the experimental protocol was initiated. Polyvinyl catheters were placed in an artery and vein of one hind leg under local anesthesia with $1 \%$ lidocaine hydrochloride. These catheters were advanced to the descending aorta and the inferior vena cava, respectively. The lambs were then anesthetized with ketamine hydrochloride (about $1 \mathrm{mg} / \mathrm{kg}$ per minute), intubated with a 4

\footnotetext{
${ }^{*}$ Gore-Tex vascular graft, registered trademark of W. L. Gore \& Associates, Inc., Newark, Del.
} 
Table I. Prebypass and postbypass arterial blood gases and hemoglobin before and after administration of nitric oxide, $100 \%$ oxygen, hypoxia, and $5 \%$ carbon dioxide ${ }^{*}$

\begin{tabular}{|c|c|c|c|c|c|c|c|c|c|c|c|c|}
\hline \multirow[b]{2}{*}{ Intervention } & \multicolumn{2}{|c|}{$p H$} & \multicolumn{2}{|c|}{$\begin{array}{c}\mathrm{PCO}_{2} \\
(\mathrm{~mm} \mathrm{Hg})\end{array}$} & \multicolumn{2}{|c|}{$\begin{array}{c}\mathrm{PO}_{2} \\
(\mathrm{~mm} \mathrm{Hg})\end{array}$} & \multicolumn{2}{|c|}{$\mathrm{HCO}_{3}$} & \multicolumn{2}{|c|}{$\begin{array}{l}\mathrm{SaO}_{2} \\
(\%)\end{array}$} & \multicolumn{2}{|c|}{$H b(g m / d l)$} \\
\hline & $\begin{array}{l}\text { Pre- } \\
C B P\end{array}$ & $\begin{array}{l}\text { Post- } \\
\text { CBP }\end{array}$ & $\begin{array}{l}\text { Pre- } \\
C B P\end{array}$ & $\begin{array}{c}\text { Post- } \\
\text { CBP }\end{array}$ & $\begin{array}{l}\text { Pre- } \\
C B P\end{array}$ & $\begin{array}{l}\text { Post- } \\
\text { CBP }\end{array}$ & $\begin{array}{l}\text { Pre- } \\
C B P\end{array}$ & $\begin{array}{l}\text { Post- } \\
C B P\end{array}$ & $\begin{array}{l}\text { Pre- } \\
C B P\end{array}$ & $\begin{array}{l}\text { Post- } \\
\text { CBP }\end{array}$ & $\begin{array}{l}\text { Pre- } \\
C B P\end{array}$ & $\begin{array}{l}\text { Post- } \\
\text { CBP }\end{array}$ \\
\hline \multicolumn{13}{|l|}{ Nitric oxide } \\
\hline \multicolumn{13}{|c|}{ Preadministration } \\
\hline Mean & 7.42 & 7.40 & 37.9 & 37.8 & 56.3 & 51.8 & 23.7 & 22.2 & 86.3 & 80.9 & 9.3 & 9.9 \\
\hline $\mathrm{SD}$ & 0.05 & 0.07 & 3.9 & 3.8 & 9.3 & 10.4 & 2.5 & 2.4 & 6.1 & 8.6 & 1.4 & 1.78 \\
\hline \multicolumn{13}{|c|}{ Postadministration } \\
\hline Mean & 7.43 & 7.41 & 33.2 & 37.7 & 61.5 & 54.3 & 23.7 & 22.9 & 90.2 & 83.2 & 9.2 & 10.0 \\
\hline $\mathrm{SD}$ & 0.04 & 0.06 & 10.8 & 3.8 & 7.3 & 10.4 & 3.0 & 2.7 & 4.1 & 6.4 & 1.4 & 1.83 \\
\hline \multicolumn{13}{|l|}{$100 \%$ Oxygen } \\
\hline \multicolumn{13}{|c|}{ Preadministration } \\
\hline Mean & 7.42 & 7.40 & 36.1 & 36.5 & 57.6 & 51.0 & 23.0 & 22.1 & 86.7 & 79.0 & 9.5 & 9.5 \\
\hline $\mathrm{SD}$ & 0.05 & 0.04 & 3.3 & 3.5 & 15.0 & 11.4 & 2.4 & 2.7 & 7.3 & 7.8 & 1.7 & 1.77 \\
\hline \multicolumn{13}{|c|}{ Postadministration } \\
\hline Mean & 7.42 & 7.37 & 36.5 & 38.7 & 295.7 & 192.0 & 23.1 & 21.8 & 89.5 & 96.4 & 9.1 & 9.5 \\
\hline $\mathrm{SD}$ & 0.07 & 0.07 & 6.7 & 6.3 & 129.7 & 105.7 & 3.1 & 2.3 & 31.1 & 4.1 & 1.5 & 1.73 \\
\hline \multicolumn{13}{|l|}{ Hypoxia } \\
\hline \multicolumn{13}{|c|}{ Preadministration } \\
\hline Mean & 7.41 & 7.39 & 37.7 & 39.2 & 56.0 & 51.7 & 23.6 & 23.1 & 78.7 & 79.6 & 9.3 & 9.8 \\
\hline $\mathrm{SD}$ & 0.06 & 0.07 & 5.1 & 5.2 & 9.0 & 11.3 & 2.8 & 1.9 & 27.7 & 10.5 & 1.6 & 1.79 \\
\hline \multicolumn{13}{|c|}{ Postadministration } \\
\hline Mean & 7.38 & 7.36 & 39.2 & 39.0 & 21.2 & 24.1 & 23.4 & 22.4 & 38.1 & 39.5 & 10.0 & 10.6 \\
\hline SD & 0.07 & 0.06 & 3.0 & 5.4 & 5.8 & 6.2 & 2.6 & 3.3 & 10.3 & 8.8 & 1.7 & 1.99 \\
\hline \multicolumn{13}{|l|}{$5 \%$ Carbon dioxide } \\
\hline \multicolumn{13}{|l|}{ Preadministration } \\
\hline Mean & 7.42 & 7.40 & 35.3 & 38.1 & 56.2 & 53.9 & 23.2 & 22.8 & 86.4 & 80.8 & 9.2 & 9.8 \\
\hline $\mathrm{SD}$ & 0.05 & 0.06 & 3.0 & 4.3 & 13.3 & 11.5 & 2.6 & 2.1 & 7.9 & 6.6 & 1.5 & 1.80 \\
\hline \multicolumn{13}{|c|}{ Postadministration } \\
\hline Mean & 7.27 & 7.25 & 53.1 & 55.5 & 51.0 & 49.2 & 24.7 & 23.4 & 79.7 & 71.5 & 9.3 & 9.9 \\
\hline $\mathrm{SD}$ & 0.05 & 0.06 & 5.3 & 5.7 & 10.8 & 9.9 & 2.5 & 2.2 & 8.1 & 6.9 & 1.6 & 1.71 \\
\hline
\end{tabular}

*Prebypass data were available for all 11 study animals, but only data from the 10 animals for which postbypass data are also available are presented in this table. $\mathrm{Hb}$, Hemoglobin; $\mathrm{HCO}_{3}$, arterial bicarbonate; $\mathrm{PCO}_{2}$, arterial partial pressure of carbon dioxide; $\mathrm{pH}$, arterial pH; $\mathrm{PO}_{2}$, arterial partial pressure of oxygen; Post-CPB, postbypass; Pre-CBP, prebypass; $\mathrm{SaO}_{2}$, arterial oxygen saturation of hemoglobin; $S D$, standard deviation.

mm outer diameter endotracheal tube, and mechanically ventilated with a Healthdyne pediatric time-cycled, pressure-limited ventilator (Healthdyne Inc., Marietta, Ga.). Pavulon (pancuronium bromide) was administered as a continuous infusion $(0.1 \mathrm{mg} / \mathrm{kg}$ intravenously). Ventilation with room air (inspired oxygen fraction 0.21) was adjusted to maintain an arterial carbon dioxide tension between 35 and $40 \mathrm{~mm} \mathrm{Hg}$. A median sternotomy incision was performed and the pericardium was incised. The patent ductus arteriosus was dissected and occluded with a vascular clip. Two single-lumen polyurethane catheters were inserted into the left and right atria, respectively. A double-lumen polyurethane catheter was placed in the right pulmonary artery. Ultrasonic flow probes (Transonic Systems Inc., Ithaca, N.Y.) were placed around the brachiocephalic trunk and the aortic arch for continuous measurement of Qs and around the $5 \mathrm{~mm}$ graft (shunt) for continuous measurement of Qp.

Data acquisition. After a 30-minute recovery following instrumentation, and before and after each intervention, arterial blood gases and hemodynamic variables were measured. Arterial blood gases and $\mathrm{pH}$ were measured on a Corning $158 \mathrm{pH} /$ blood gas analyzer (Corning Medical and Scientific, Medfield, Mass.), and hemoglobin concentration and oxygen saturation were measured by a hemoximeter (model OSM 2; Radiometer A/S, Copenhagen, Denmark). Pulmonary and systemic arterial pressures (PAP and SAP, respectively) and right and left atrial pressures were measured with Statham P23Db pressure transducers (Statham Instruments, Hato Rey, Puerto Rico). Mean pressures were obtained by electrical integration. Heart rate was measured by a cardiotachymeter triggered from the phasic systemic arterial pressure pulse wave. $\mathrm{Qp}$ and $\mathrm{Qs}$ were measured on an ultrasonic flowmeter (Transonic Systems Inc.). All hemodynamic variables were continuously recorded on a Gould multichannel electrostatic recorder (Gould Inc., Cleveland, Ohio). PVR and SVR were calculated by standard formulas relating pressure to flow and resistance (Ohm's law).

Experimental protocol. After baseline measurements of the hemodynamic variables (PAP, SAP, heart rate, Qs, $\mathrm{Qp}$, and left and right atrial pressures) and systemic 
Table II. Hemodynamic effects of nitric oxide, $100 \%$ oxygen, hypoxia, and $5 \%$ carbon dioxide in single ventricle physiology

\begin{tabular}{|c|c|c|c|c|c|c|c|c|}
\hline & \multicolumn{2}{|c|}{ Nitric oxide } & \multicolumn{2}{|c|}{$100 \%$ Oxygen } & \multicolumn{2}{|c|}{ Hypoxia } & \multicolumn{2}{|c|}{$5 \%$ Carbon dioxide } \\
\hline & Before & With & Before & With & Before & With & Before & With \\
\hline \multicolumn{9}{|l|}{ PAP } \\
\hline Prebypass & $28.4 \pm 5.7$ & $24.8 \pm 5^{*}$ & $29.6 \pm 4.5$ & $28 \pm 4.2^{*}$ & $29.5 \pm 4.4$ & $35.1 \pm 4.4^{*}$ & $28.2 \pm 5.3$ & $32 \pm 6.6^{*}$ \\
\hline Postbypass & $29.1 \pm 3.8$ & $26.2 \pm 4.2^{*}$ & $30.8 \pm 5.2$ & $28.6 \pm 5.5^{*}$ & $29.6 \pm 6.4$ & $36.4 \pm 7.6^{*}$ & $28.4 \pm 6$ & $32.7 \pm 6.3^{*}$ \\
\hline \multicolumn{9}{|l|}{ SAP } \\
\hline Prebypass & $53.3 \pm 11.9$ & $50.5 \pm 12.3^{*}$ & $55.5 \pm 9$ & $56.1 \pm 8.8$ & $56.5 \pm 10$ & $50.5 \pm 9.7^{*}$ & $53.1 \pm 9.5$ & $52.9 \pm 9$ \\
\hline Postbypass & $52.9 \pm 9.5$ & $50.2 \pm 8.8^{*}$ & $55.4 \pm 7.5$ & $56.4 \pm 7.6$ & $54.2 \pm 11.8$ & $48.4 \pm 14.1^{*}$ & $52.4 \pm 8.5$ & $51.2 \pm 8.2$ \\
\hline \multicolumn{9}{|l|}{ Qp } \\
\hline Prebypass & $231 \pm 43$ & $238 \pm 45^{*}$ & $231 \pm 47$ & $269 \pm 51^{*}$ & $232 \pm 61$ & $189 \pm 52^{*}$ & $235 \pm 50$ & $197 \pm 47^{*}$ \\
\hline Postbypass & $213 \pm 60$ & $218.7 \pm 62^{*}$ & $227 \pm 57$ & $257 \pm 52^{*}$ & $233 \pm 62$ & $190 \pm 56^{*}$ & $216 \pm 66$ & $185 \pm 59^{*}$ \\
\hline \multicolumn{9}{|l|}{ Qs } \\
\hline Prebypass & $122 \pm 44$ & $117 \pm 43^{*}$ & $133 \pm 55$ & $120 \pm 52^{*}$ & $135 \pm 42$ & $161 \pm 47^{*}$ & $125 \pm 30$ & $142 \pm 32^{*}$ \\
\hline Postbypass & $164 \pm 57$ & $162 \pm 56^{*}$ & $148 \pm 40$ & $141 \pm 42^{*}$ & $150 \pm 53$ & $166 \pm 52^{*}$ & $156 \pm 55$ & $180 \pm 58^{*}$ \\
\hline \multicolumn{9}{|l|}{$\mathrm{Qp} / \mathrm{Qs}$} \\
\hline Prebypass & $2.04 \pm 0.66$ & $2.18 \pm 0.67^{*}$ & $1.85 \pm 0.51$ & $2.56 \pm 0.99^{*}$ & $1.80 \pm 0.61$ & $1.19 \pm 0.35^{*}$ & $1.94 \pm 0.46$ & $1.39 \pm 0.34^{*}$ \\
\hline Postbypass & $1.45 \pm 0.63$ & $1.50 \pm 0.65^{*}$ & $1.64 \pm 0.55$ & $2.02 \pm 0.82^{*}$ & $1.78 \pm 0.82$ & $1.27 \pm 0.55^{*}$ & $1.54 \pm 0.67$ & $1.11 \pm 0.47^{*}$ \\
\hline \multicolumn{9}{|c|}{ PVR } \\
\hline Prebypass & $0.127 \pm 0.036$ & $0.109 \pm 0.034^{*}$ & $0.136 \pm 0.042$ & $0.108 \pm 0.029^{*}$ & $0.134 \pm 0.035$ & $0.202 \pm 0.073^{*}$ & $0.124 \pm 0.029$ & $0.167 \pm 0.041^{*}$ \\
\hline Postbypass & $0.148 \pm 0.047$ & $0.131 \pm 0.050^{*}$ & $0.142 \pm 0.036$ & $0.115 \pm 0.026^{*}$ & $0.134 \pm 0.043$ & $0.203 \pm 0.057^{*}$ & $0.141 \pm 0.046$ & $0.191 \pm 0.060^{*}$ \\
\hline \multicolumn{9}{|l|}{ SVR } \\
\hline Prebypass & $0.504 \pm 0.254$ & $0.494 \pm 0.248^{*}$ & $0.472 \pm 0.190$ & $0.559 \pm 0.292^{*}$ & $0.473 \pm 0.211$ & $0.344 \pm 0.137^{*}$ & $0.461 \pm 0.182$ & $0.399 \pm 0.148^{*}$ \\
\hline Postbypass & $0.385 \pm 0.212$ & $0.368 \pm 0.193^{*}$ & $0.406 \pm 0.153$ & $0.452 \pm 0.208^{*}$ & $0.419 \pm 0.229$ & $0.327 \pm 0.184^{*}$ & $0.389 \pm 0.176$ & $0.320 \pm 0.129^{*}$ \\
\hline
\end{tabular}

$P A P$, Pulmonary artery pressure $(\mathrm{mm} \mathrm{Hg}) ; S A P$, systemic arterial pressure $(\mathrm{mm} \mathrm{Hg}) ; Q p$, pulmonary blood flow $(\mathrm{ml} / \mathrm{kg} / \mathrm{min}) ; Q s$, systemic blood flow $(\mathrm{ml} / \mathrm{kg} / \mathrm{min}) ; P V R$, pulmonary vascular resistance $\left(\mathrm{mm} \mathrm{Hg} \cdot\left[\mathrm{ml} / \mathrm{min}^{\prime} / \mathrm{kg}\right]^{-1}\right) ; S V R$, systemic vascular resistance $\left(\mathrm{mm} \mathrm{Hg} \cdot(\mathrm{ml} / \mathrm{min} / \mathrm{kg})^{-1}\right)$.

*The change in the parameter measured during intervention (oxygen, nitric oxide, carbon dioxide, and hypoxia) was statistically significant ( $p \leq 0.05$ ).

arterial blood gases, the following inhalational agents were administered in random order and their effects were noted:

Oxygen $100 \%$. A $100 \%$ concentration of oxygen was administered by increasing the inspired oxygen fraction on the ventilator to $100 \%$ (oxygen concentration was checked with an oxygen sensor in the inflow circuit).

Oxygen 10\% (hypoxia). A $10 \%$ concentration of oxygen was administered by adding nitrogen to the inspired air (oxygen concentration was checked with an oxygen sensor in the inflow circuit).

Carbon dioxide 5\%. A commercially prepared gas mixture of $5 \%$ carbon dioxide, $21 \%$ oxygen, and $74 \%$ nitrogen was administered.

Nitric oxide 80 parts per million. Nitric oxide was administered into the inflow circuit of the ventilator. The flow of the nitric oxide-nitrogen mixture was regulated by a Bird low-flow blender (Bird Products Corp., Palm Springs, Calif.), and delivered nitric oxide concentration ( $80 \mathrm{ppm}$ of air) was continuously measured at the endotracheal tube by chemiluminescence (model 42H; Thermo-Environmental Instruments, Franklin, Mass.).

After administration of $100 \%$ oxygen and $5 \%$ carbon dioxide, a 10 -minute period was provided for stabilization. Hemodynamic variables were measured continuously. Systemic arterial blood gases, $\mathrm{pH}$, and hemodynamic variables were measured at the end of 10 minutes. After administration of $10 \%$ oxygen and nitric oxide, a 5-minute period of stabilization was allowed and the same variables were recorded. At the completion of each intervention, a 30 -minute recovery period was allowed before the next intervention, and all measurements were repeated to ensure that the animal was in a steady state.

The lambs were then supported with cardiopulmonary bypass, cooled to $15^{\circ} \mathrm{C}$, and the circulation was arrested for 30 minutes to mimic the clinical circumstances of a stage I Norwood procedure. The lambs were rewarmed fully and separated from bypass. After a 60 -minute recovery period, all hemodynamic measurements and systemic arterial blood gases and $\mathrm{pH}$ levels were obtained and a stable state was established. The prebypass interventions were repeated randomly according to the same protocol. At the end of the study, the lambs were given a lethal dose of pentobarbital sodium and an autopsy was performed to confirm the placement of intravascular catheters and shunt patency. All animals received humane care in compliance with the "Principles of Laboratory Animal Care" formulated by the National Society of Medical Research and the "Guide for the Care and Use of Laboratory Animals" prepared by the National Academy of Sciences and published by the National Institutes of Health (NIH Publication No. 86-23, revised 1985). All protocols were approved by the Committee on Animal Research of the University of California, San Francisco.

Statistical analysis. Microsoft Excel Version 5.0 (Microsoft Corporation, Redmond, Wash.) was used to calculate descriptive and analytic statistics. The change in the variables with each intervention is expressed as percent 


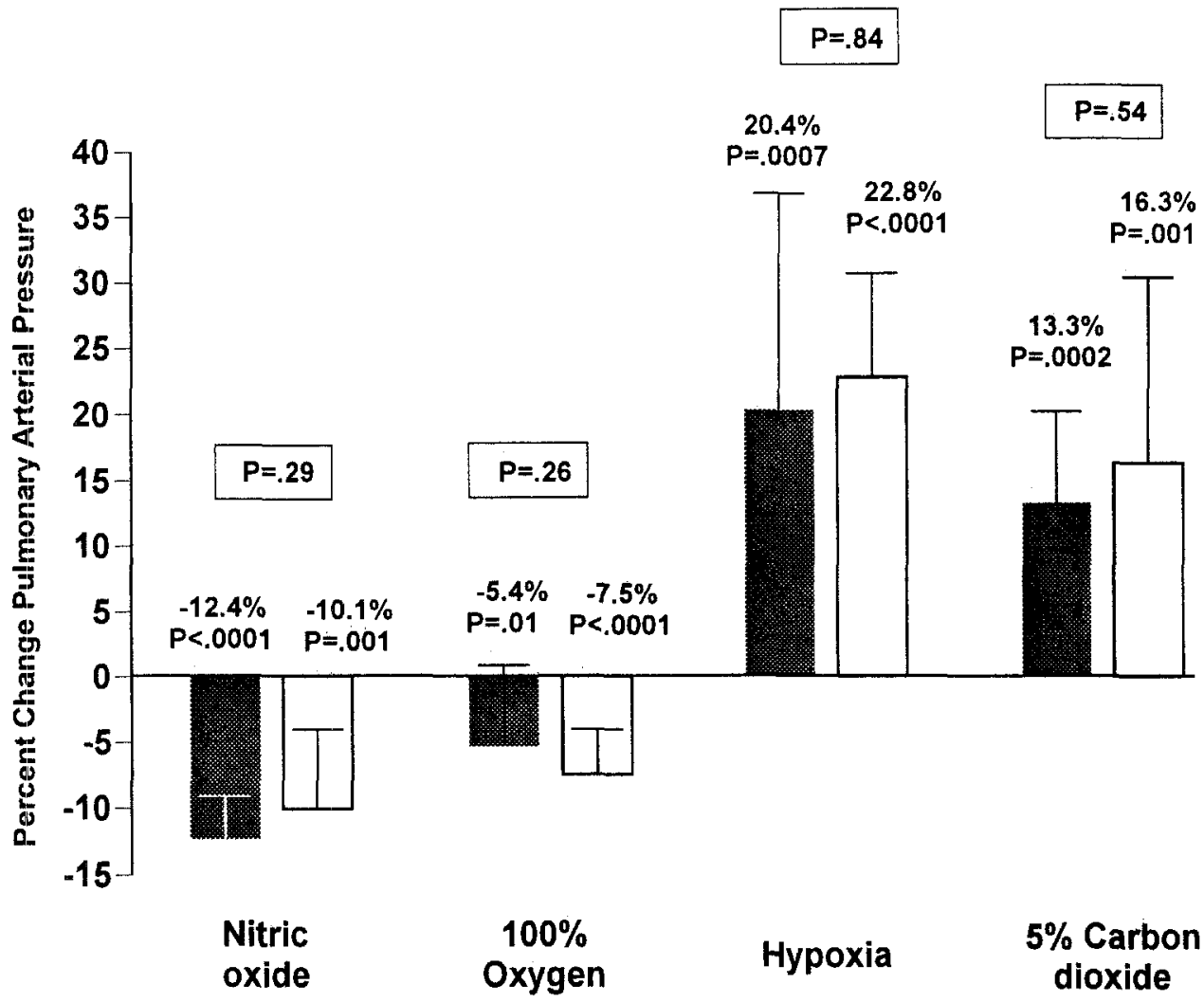

\section{Pre-Bypass}

\section{Post-Bypass}

Fig. 2. Mean ( \pm standard deviation) percent change in PAP after administration of nitric oxide, $100 \%$ oxygen, hypoxia, and 5\% carbon dioxide before $(n=11)$ and after $(n=10)$ cardiopulmonary bypass. Percent and $p$ values displayed directly above figure columns reflect the mean percent change and significance levels for the paired, two-tailed $t$ test comparison between preadministration and postadministration PAP. Boxed $p$ values indicate significance levels for paired two-tailed $t$ test comparison between prebypass and postbypass mean percent change in PAP after administration of nitric oxide, $100 \%$ oxygen, hypoxia, and 5\% carbon dioxide $(n=10)$. The same format applies to Figs. 3 through 9.

change from the baseline preintervention value. Mean percent changes in variables from preadministration to postadministration levels (as presented in the Results section) were calculated as the average of percent changes in individual subjects. Paired two-tailed $t$ test analysis was used to assess the significance of changes in hemodynamic variables relative to baseline preadministration levels after nitric oxide, $100 \%$ oxygen, $10 \%$ oxygen, and $5 \%$ carbon dioxide administration, both before and after bypass. The significance of differences between prebypass and postbypass mean percent change in variables after the administration of nitric oxide, $100 \%$ oxygen, $10 \%$ oxygen, and $5 \%$ carbon dioxide was also examined by means of paired two-tailed $t$ test analysis. In all cases, presented $p$ values reflect significance levels by two-tailed paired $t$ test comparison, as described earlier. All $p$ values of 0.05 or less were considered significant.

\section{Results}

Of the 14 fetuses in which the model was created, 11 were delivered normally and three were aborted within 3 days of the in utero operation. All animals remained in hemodynamically stable condition during the prebypass study, and hemodynamic and blood gas data were obtained and analyzed in all 11 animals studied. The study was discontinued in one lamb because of excessive bleeding from rupture of the graft suture line during cannulation of the ascending aorta. The other 10 animals were in hemodynamically stable condition throughout the bypass and postbypass periods and completed the study successfully. Except for postbypass nitric oxide 


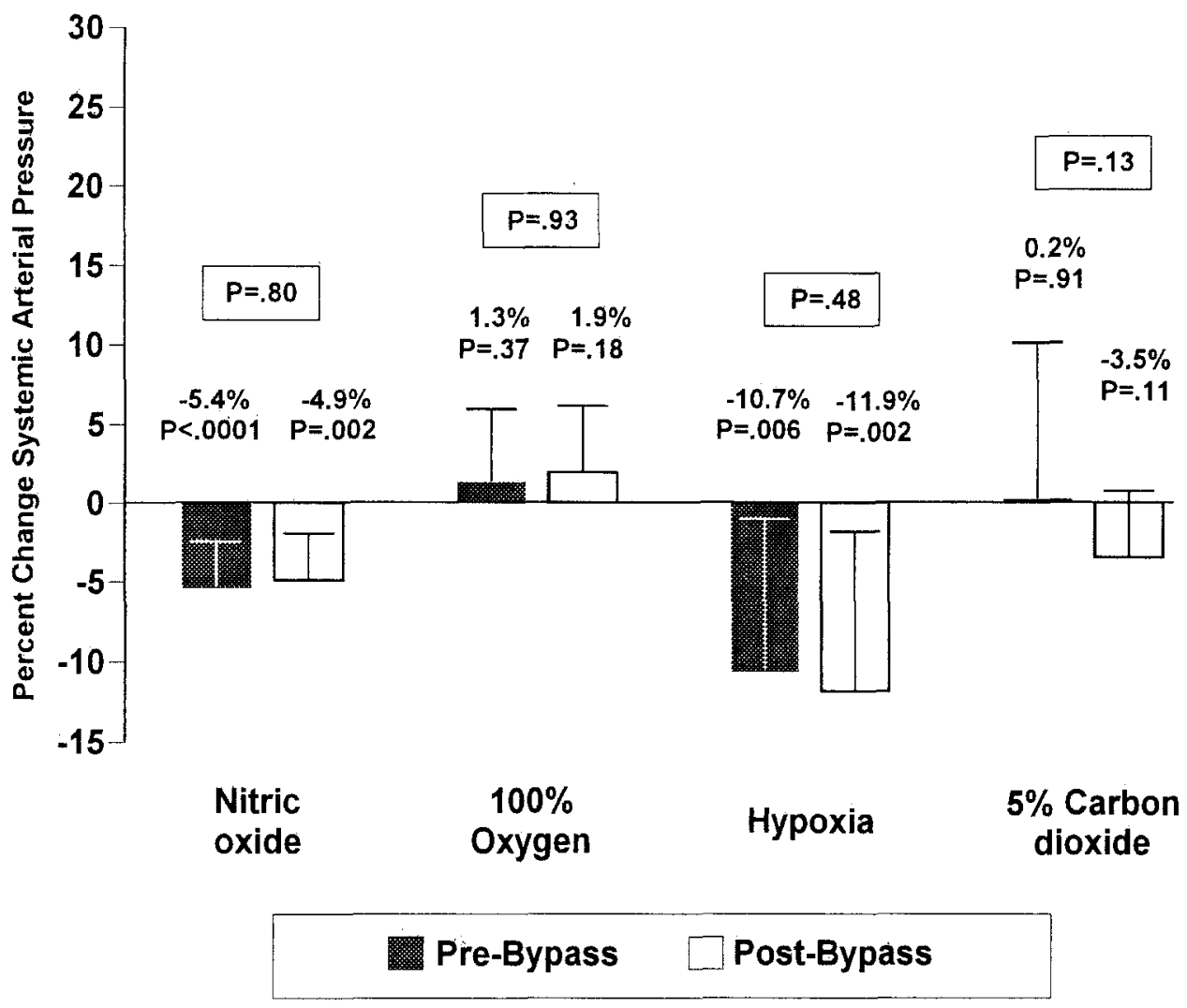

Fig. 3. Mean ( \pm standard deviation) percent change in SAP after administration of nitric oxide, $100 \%$ oxygen, hypoxia, and $5 \%$ carbon dioxide before $(n=11)$ and after $(n=9)$ cardiopulmonary bypass.

data, which were not obtained for technical reasons in the first animal studied, postbypass hemodynamic and blood gas data were available for all 10 animals. Prebypass changes after nitric oxide, 100\% oxygen, hypoxia, and $5 \%$ carbon dioxide administration were calculated from the data collected on all 11 animals. Postbypass statistics (except in the case of nitric oxide) reflect data for the 10 animals that survived bypass. Paired two-tailed $t$ test comparison of prebypass and postbypass changes after intervention was performed with the use of data from the 10 animals that survived bypass and from nine animals for nitric oxide.

Arterial blood gases and hemoglobin. Table I shows arterial $\mathrm{pH}$, carbon dioxide tension, oxygen tension, bicarbonate, oxygen saturation, and hemoglobin levels before and after administration of nitric oxide, $100 \%$ oxygen, hypoxia; and $5 \%$ carbon dioxide before and after bypass.

Hemodynamic data. The absolute values of hemodynamic responses to nitric oxide, $100 \%$ oxygen, hypoxia, and $5 \%$ carbon dioxide are represented in Table II and are summarized briefly in the following paragraphs.

$P A P$ (Fig. 2). Nitric oxide before bypass resulted in a fall in PAP $(-12.4 \% ; p<0.0001)$. Nitric oxide infusion after bypass also caused a decrease in PAP $(-10.1 \% ; p=0.001)$. Administration of $100 \%$ oxygen led to a drop in PAP before bypass $(-5.4 \% ; p=$ $0.01)$ and after bypass $(-7.5 \% ; p<0.0001)$. Prebypass hypoxia produced a significant rise in PAP $(20.4 \% ; p=0.0007)$, as did postbypass hypoxia (22.8\%; $p<0.0001)$. Administration of $5 \%$ carbon dioxide produced a significant increase in PAP before bypass $(13.3 \% ; p=0.0002)$ and after bypass $(16.3 \% ; p=0.001)$.

$S A P$ (Fig. 3). Administration of nitric oxide produced in a small but consistent drop in SAP before bypass $(-5.4 \% ; p<0.0001)$ and after bypass $(-4.9 \% ; p=0.002)$. There were no significant changes in SAP with $100 \%$ oxygen administration. Prebypass and postbypass hypoxia led to significant 


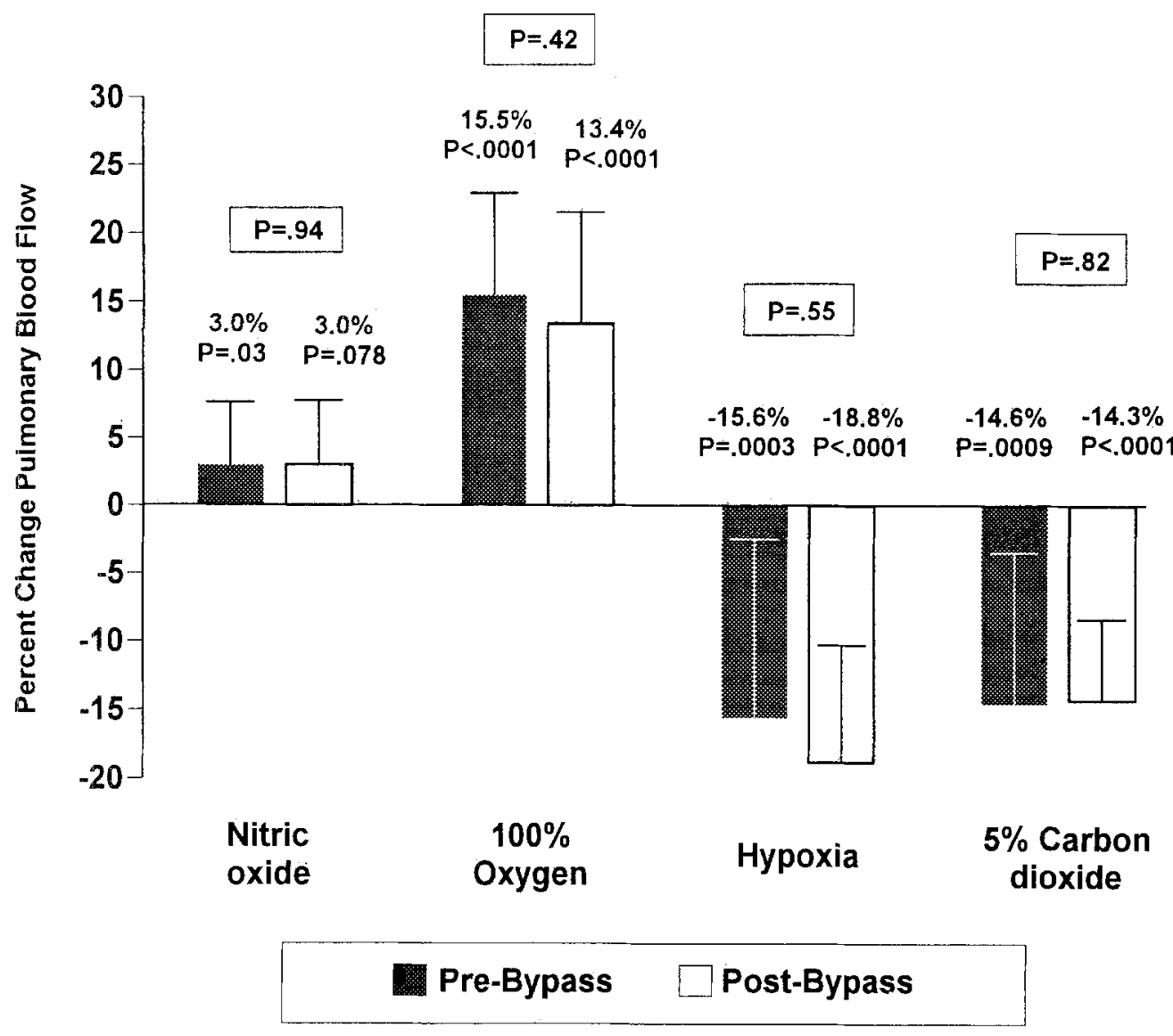

Fig. 4. Mean ( \pm standard deviation) percent change in indexed Qp after administration of nitric oxide, $100 \%$ oxygen, hypoxia, and $5 \%$ carbon dioxide before $(n=11)$ and after $(n=10)$ cardiopulmonary bypass.

decreases in SAP (prebypass: $-10.7 \% ; p=0.006$; postbypass: $-11.9 \% ; p=0.002$ ). Administration of $5 \%$ carbon dioxide had almost no effect on SAP before or after bypass.

$Q p$ (Fig. 4). Qp increased by $3.0 \%$ after nitric oxide administration both before and after bypass. Large and significant increases in Qp resulted from $100 \%$ oxygen administration before $(15.5 \% ; p<$ $0.0001)$ and after bypass $(13.4 \% ; p<0.0001)$. Increases in Qp caused by $100 \%$ oxygen were significantly greater than those caused by nitric oxide before bypass $(p=0.001)$ and after bypass $(p=$ 0.012). Hypoxia before bypass led to a significant fall in Qp $(-15.6 \% ; p=0.0003)$, as did postbypass hypoxia $(-18.8 \% ; p<0.0001)$. The effect of $5 \%$ carbon dioxide administration on Qp was similar to that of hypoxia, with similar and significant prebypass $(-14.6 \% ; p=0.0009)$ and postbypass decreases $(-14.3 \% ; p<0.0001)$. There was no signif- icant difference in the effects on Qp of hypoxia and $5 \%$ carbon dioxide between prebypass $(p=0.84)$ or postbypass states $(p=0.19)$.

Qs (Fig. 5). Small but consistent decreases in Qs were noted after nitric oxide administration before bypass $(-3.5 \% ; p=0.02)$ and after bypass $(-1.4 \%$; $p=0.03$ ). Administration of $100 \%$ oxygen also led to significant decreases in prebypass Qs $(-11.0 \%$; $p=0.0004)$ and postbypass Qs $(-6.2 \% ; p=0.01)$. Qs increased significantly after prebypass hypoxia $(24.0 \% ; p=0.002)$ and postbypass hypoxia $(12.3 \%$; $p=0.012$ ). Qs rose significantly after prebypass $(15.4 \% ; p=0.002)$ and postbypass administration of $5 \%$ carbon dioxide $(17.3 \% ; p=0.0008)$.

Qp/Qs ratio (Fig. 6). Nitric oxide increased Qp/Qs ratio by $7.1 \%(p=0.014)$ before bypass and to a lesser degree after bypass $(4.2 \% ; p=0.05)$. However, $100 \%$ oxygen produced a significant increase in Qp/Qs ratio both before and after bypass (preby- 


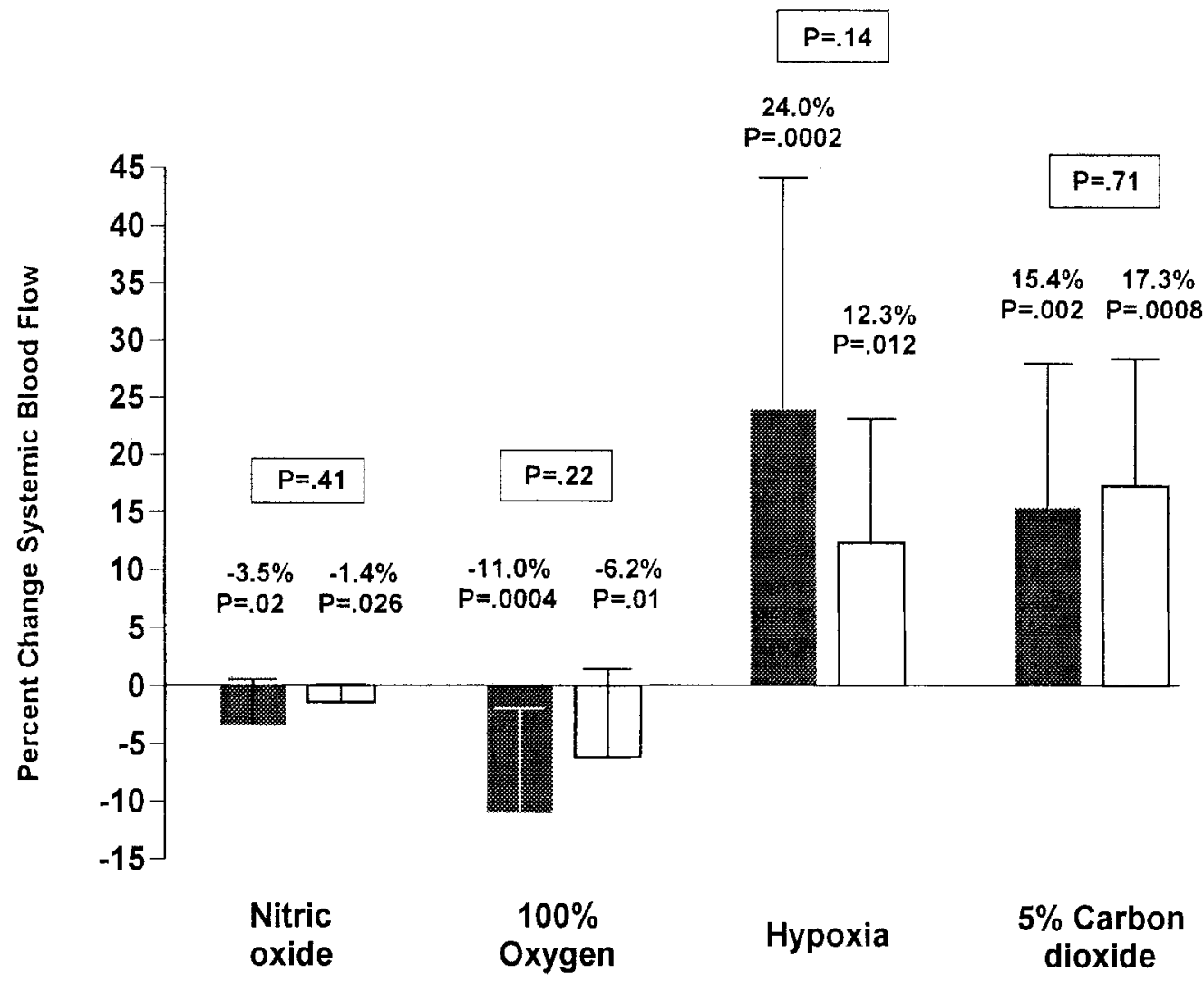

\section{Pre-Bypass $\square$ Post-Bypass}

Fig. 5. Mean ( \pm standard deviation) percent change in indexed Qs after administration of nitric oxide, $100 \%$ oxygen, hypoxia, and $5 \%$ carbon dioxide before $(n=11)$ and after $(n=10)$ cardiopulmonary bypass.

pass: $36.6 \% ; p=0.001$; postbypass: $21.7 \% ; p=$ $0.01)$. Qp/Qs ratio increased significantly more with $100 \%$ oxygen than with nitric oxide before $(p=$ $0.008)$ and after bypass $(p=0.009)$. A significant fall in $\mathrm{Qp} / \mathrm{Qs}$ ratio was seen with hypoxia (prebypass: $-32.2 \% ; p<0.0001$; postbypass: $-27.4 \% ; p=$ 0.0003 ) and $5 \%$ carbon dioxide (prebypass: $-26.9 \%$; $p=0.001$; postbypass: $-26.2 \% ; p=0.0009$ ). The effects of hypoxia and $5 \%$ carbon dioxide were not significantly different before $(p=0.28)$ or after bypass $(p=0.63)$.

PVR (Fig. 7). PVR decreased after nitric oxide administration before $(-14.8 \% ; p<0.0001)$ and after bypass $(-12.1 \% ; p=0.01)$. Similarly, PVR dropped significantly after $100 \%$ oxygen administration before $(-19.0 \% ; p=0.0005)$ and after bypass $(-18.1 \% ; p=0.0002)$. A large and significant rise in PVR followed prebypass hypoxia $(47.9 \% ; p=$
$0.0003)$ and postbypass hypoxia $(53.9 \% ; p<$ 0.0001 ), as well as administration of $5 \%$ carbon dioxide before $(34.7 \% ; p<0.0001)$ and after bypass $(35.7 \% ; p<0.0001)$. The increase in PVR caused by hypoxia was not significantly greater than that caused by $5 \%$ carbon dioxide before bypass $(p=$ $0.08)$, but it was significantly greater after bypass $(p=0.03)$.

SVR (Fig. 8). Nitric oxide administration produced a small but consistent decrease in SVR (prebypass: $-2.0 \%, p=0.05 ;-3.6 \%, p=0.07$ ). Administration of $100 \%$ oxygen led to an increase in SVR before $(15.0 \% ; p=0.036)$ and after bypass $(9.0 \% ; p=0.03)$. With hypoxia, SVR decreased substantially both before bypass $(-25.5 \% ; p=$ $0.003)$ and after bypass $(-22.2 \% ; p=0.0006)$. After prebypass and postbypass administration of $5 \%$ carbon dioxide, the SVR decreased (prebypass: 


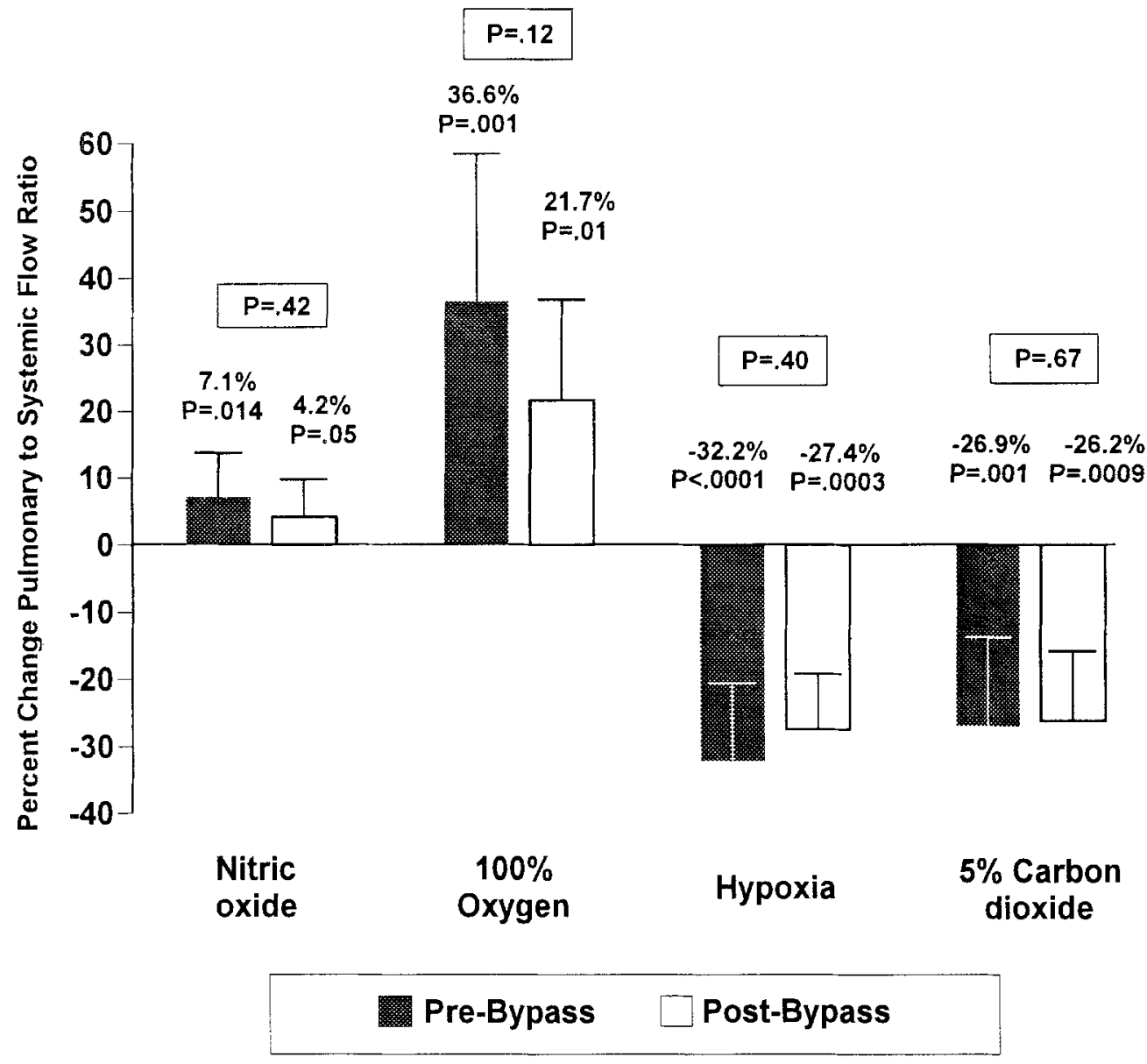

Fig. 6. Mean ( \pm standard deviation) percent change in Qp/Qs after administration of nitric oxide, $100 \%$ oxygen, hypoxia, and $5 \%$ carbon dioxide before $(n=11)$ and after $(n=10)$ cardiopulmonary bypass.

$-12.3 \% ; p=0.016 ;$ postbypass: $-15.9 \% ; p=$ $0.005)$.

PVR/SVR ratio (Fig, 9). PVR/SVR ratio after prebypass nitric oxide decreased by $13.0 \%(p=$ 0.001 ), and a similar response was observed when nitric oxide was given after bypass $(-8.8 \% ; p=$ 0.038 ). Administration of $100 \%$ oxygen produced a significant drop in PVR/SVR ratio (prebypass: $-28.6 \% ; p=0.0001$; postbypass: $-25.4 \% ; p=$ $0.0006)$. PVR/SVR ratio dropped significantly more with $100 \%$ oxygen than it did with nitric oxide before bypass $(p=0.007)$ and after bypass $(p=$ $0.009)$. PVR/SVR ratio approximately doubled after both prebypass $(104.5 \% ; p<0.0001)$ and postbypass hypoxia $(96.9 \% ; p=0.003)$. Large and significant increases in PVR/SVR ratio also followed 5\% carbon dioxide administration both before bypass $(57.7 \% ; p=0.0003)$ and after bypass $(58.6 \% ; p=$
0.0001). The hypoxia-induced increase in PVR/SVR ratio was greater than the increase caused by $5 \%$ carbon dioxide (prebypass: $p=0.04$; postbypass: $p=0.065$ ).

\section{Discussion}

Perioperative management of patients born with single ventricle physiology is aimed at achieving a critical balance between $\mathrm{Op}$ and Qs to ensure adequate systemic oxygen delivery while simultaneously minimizing volume overload on the single functioning ventricle. ${ }^{1,2}$ Clinical strategies to achieve this goal are often institutional preferences, which are generally based on experience with blood gas measurements and final patient outcome. ${ }^{10-12}$ This approach tends to create controversy because institutions with different approaches ${ }^{10,12}$ often have equally good results. Frequently, other factors such as ventricular 


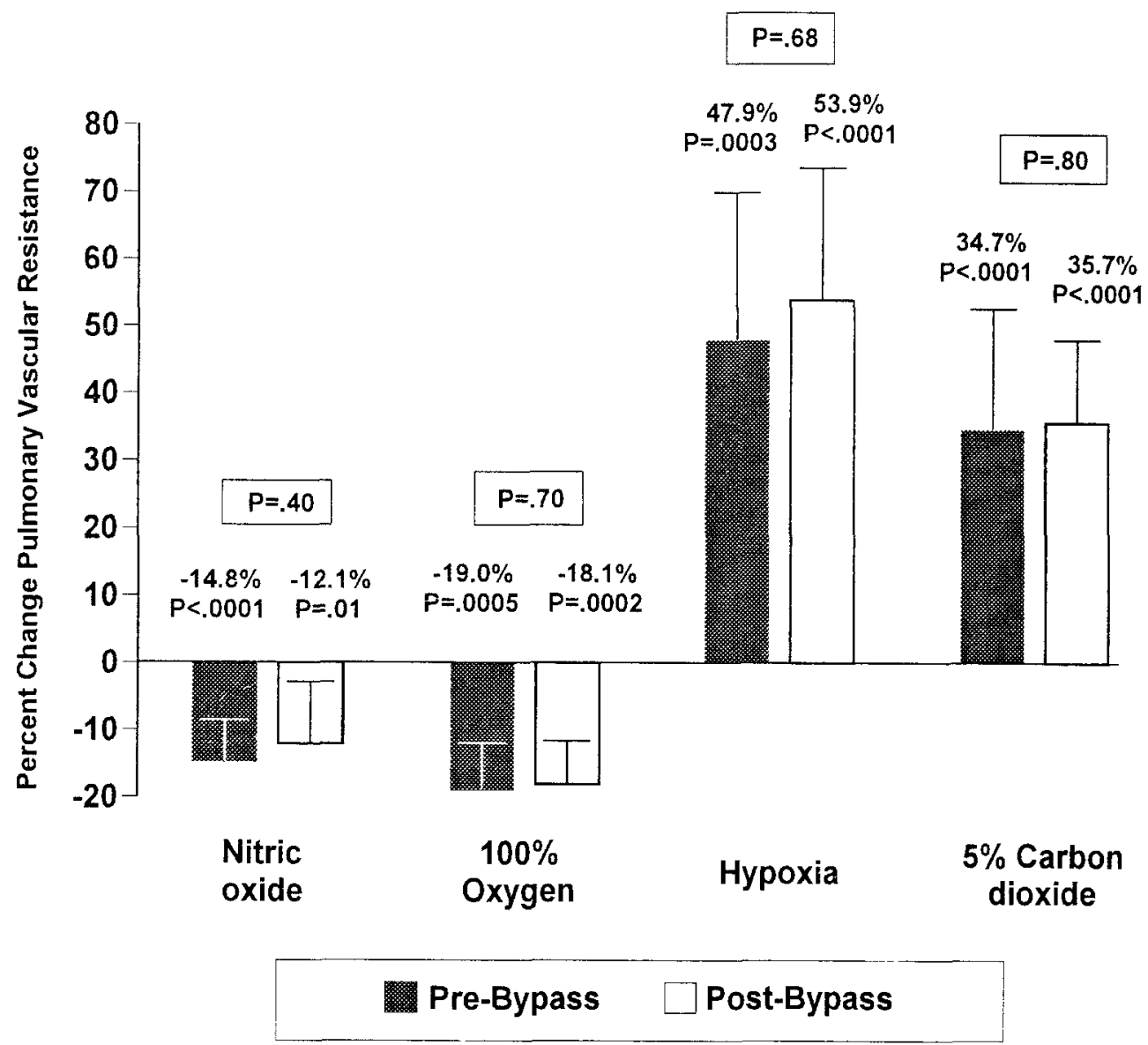

Fig. 7. Mean ( \pm standard deviation) percent change in PVR after administration of nitric oxide, $100 \%$ oxygen, hypoxia, and $5 \%$ carbon dioxide before $(n=11)$ and after $(n=10)$ cardiopulmonary bypass.

function and the size of the systemic-pulmonary shunt are not taken into consideration. This empiric nature of current management strategies has prompted investigators to create animal models of single ventricle physiology to investigate the hemodynamic effects of various interventions by direct methods. Recently, Mora and associates ${ }^{13}$ reported an experimental model of single ventricle physiology in which they examined the effects of inspired carbon dioxide on pulmonary hemodynamics. This study supported the clinical use of inspired carbon dioxide to manipulate PVR and decrease the Qp/Qs ratio. However, one drawback of the reported model is that it was created in a 4-week-old animal that had progressed beyond the transitional phase of circulation. As such, it had lived for a considerable period with normal postnatal circulatory dynamics before it was surgically altered to mimic single ventricle physiology. Thus this model does not accurately represent a neonate with single ventricle physiology who requires intervention in the first few days of life.

We recently created a fetal model of single ventricle physiology. The main shortcoming of this model is that it has two ventricles, both of which eject into the aorta, where the mixing of systemic and pulmonary venous blood occurs. In addition, inherent to any animal model is the possibility of differences in physiologic responses between human beings and animals. Inasmuch as the perinatal circulatory physiology has been most extensively studied in the sheep, and the size of the sheep fetus is similar to that of the human fetus, we preferred the sheep fetus for the model. However, the major strength of this model is that the most important feature of single ventricle physiology, namely, parallel pulmonary and systemic circulations, is present from birth, which serves to adequately simulate the newborn infant with a single ventricle. This fetal model allows 


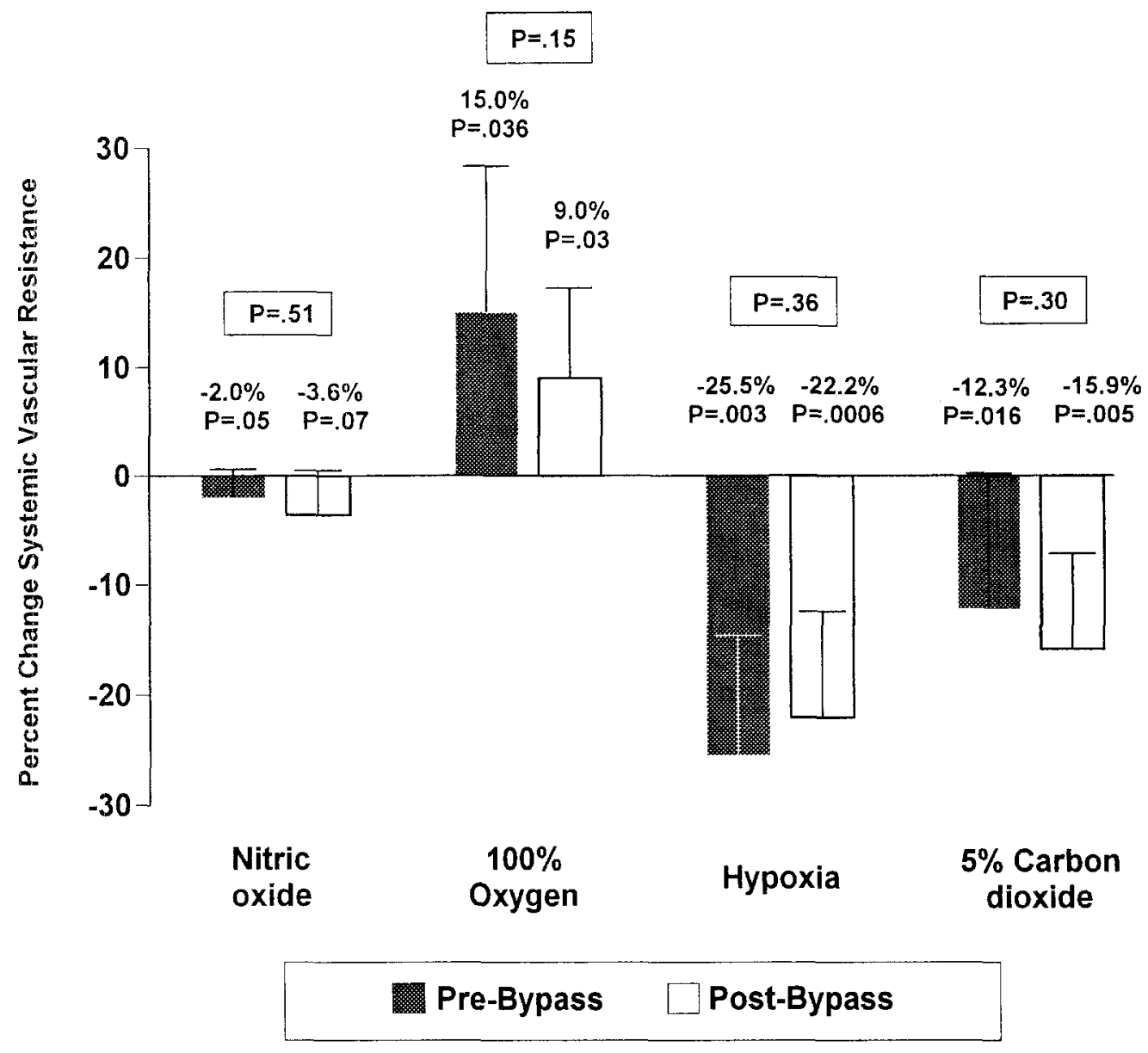

Fig. 8. Mean ( \pm standard deviation) percent change in SVR after administration of nitric oxide, $100 \%$ oxygen, hypoxia, and $5 \%$ carbon dioxide before $(n=11)$ and after $(n=10)$ cardiopulmonary bypass.

for the study of single ventricle physiology during the transitional period when most neonates undergo surgical intervention.

In the present study, as expected, $100 \%$ oxygen and nitric oxide both decreased PVR and increased Qp/Qs ratio. PVR decreased approximately $20 \%$ with oxygen and $12 \%$ to $14 \%$ with nitric oxide. The magnitude of increase in Qp/Qs ratio was significantly greater with oxygen than with nitric oxide $(p=0.008$ before bypass and $p=0.009$ after bypass). This difference in the effect on $Q p / Q$ s ratio is probably due to the observed differences in the effect of $100 \%$ oxygen and nitric oxide on SVR. Nitric oxide decreased SVR, a phenomenon that has also been observed by others ${ }^{15}$ in the setting of single ventricle physiology. However, its effect is difficult to explain, because nitric oxide is inactivated by contact with hemoglobin and thought not to have any systemic effects. On the other hand, the increase in SVR with oxygen appears to be a flow-related phenomenon resulting from steal of systemic flow into the pulmonary bed. The increase in $\mathrm{Qp} / \mathrm{Qs}$ ratio with $100 \%$ oxygen is more profound than with 80 ppm nitric oxide. Nitric oxide may be useful when a mild increase in $Q p / Q s$ ratio is adequate to achieve systemic oxygenation.

Both carbon dioxide and hypoxia (10\% oxygen) increased PVR and decreased both $Q p$ and the $\mathrm{Qp} / \mathrm{Qs}$ ratio. The effects of hypoxia were significantly more pronounced than those of carbon dioxide on PVR, especially after bypass, but there was no statistically significant difference between decreases in $\mathrm{Qp} / \mathrm{Qs}$ ratio produced by carbon dioxide and hypoxia. Both hypoxia and carbon dioxide decreased SVR. However, this effect could be flow related as a result of a reciprocal increase in Qs 


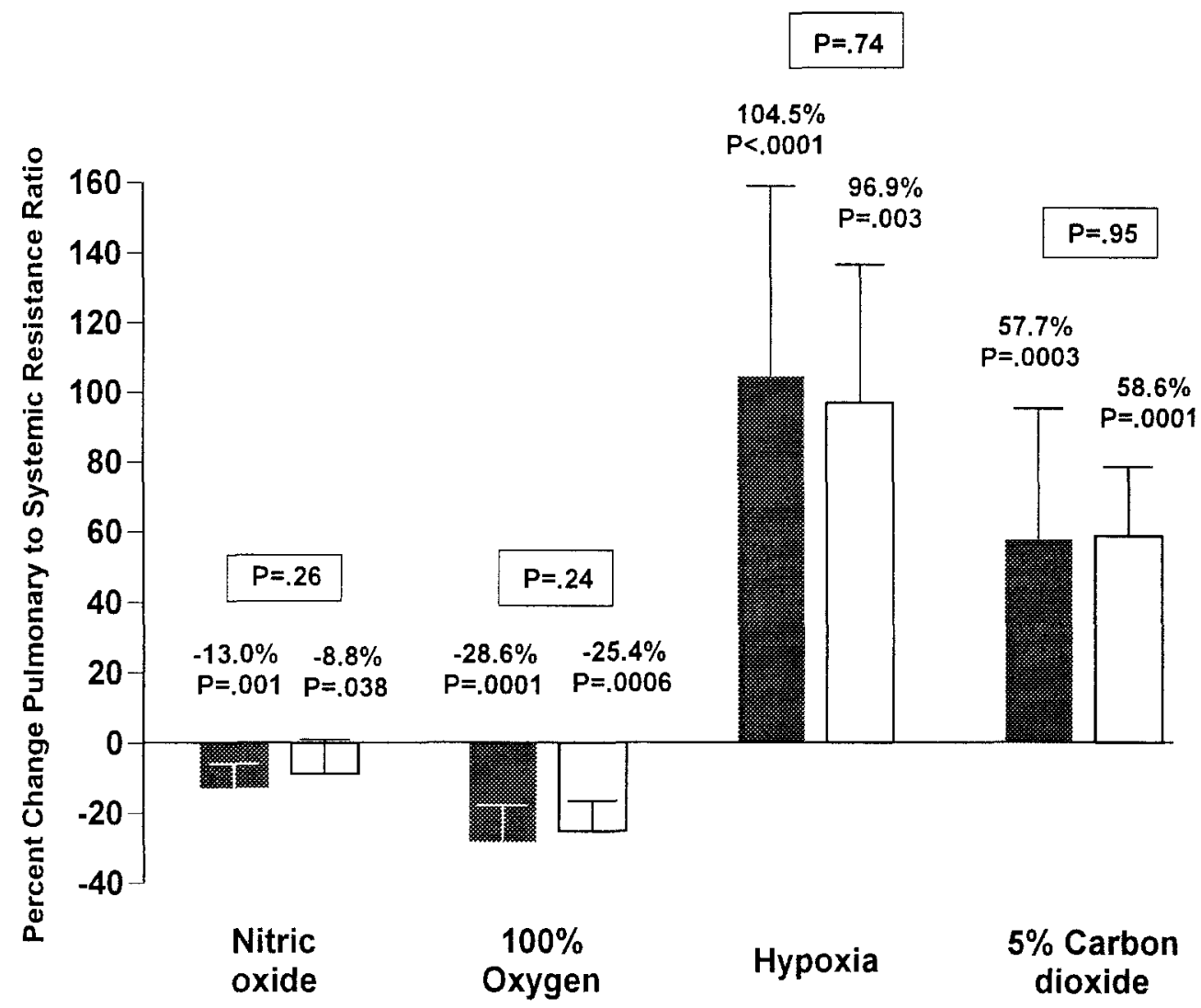

- Pre-Bypass $\square$ Post-Bypass

Fig. 9. Mean ( \pm standard deviation) percent change in PVR/SVR after administration of nitric oxide, $100 \%$ oxygen, hypoxia, and 5\% carbon dioxide before $(n=11)$ and after $(n=10)$ cardiopulmonary bypass.

caused by elevated PVR. The effects of carbon dioxide in this study were not measured independent of $\mathrm{pH}$. However, a previous study in a model of single ventricle suggests that carbon dioxide may have independent effects on pulmonary vasculature when $\mathrm{pH}$ is controlled. ${ }^{13}$

In summary, judicious use of oxygen, nitric oxide, and carbon dioxide all appear to be useful modalities to manipulate PVR and alter Qp/Qs ratio. The model described here offers a unique tool to study single ventricle physiology. Further studies to quantitate the pulmonary vascular responses to incremental changes in inspired gases and their effects on systemic oxygen delivery will be necessary for a better understanding of single ventricle physiology in the neonatal transitional period and after bypass.
We thank Roger Chang for his technical help in conducting these studies.

\section{REFERENCES}

1. Castaneda AR, Jonas RA, Mayer JE, Hanley FL, editors. Cardiac surgery of the neonate and infant. Philadelphia: WB Saunders, 1994:80-2.

2. Jobes DR, Nicolson SC, Steven JM, Miller M, Jacobs ML, Norwood WI. Carbon dioxide prevents pulmonary overcirculation in hypoplastic left heart syndrome. Ann Thorac Surg 1992;54:150-1.

3. Roberts JD, Lanf P, Bigatello LM, Vlahakes GJ, Zapol WM. Inhaled nitric oxide in congenital heart disease. Circulation 1993;87:447-52.

4. Rudolph AM, Yuan S. Response of the pulmonary vasculature to hypoxia and hydrogen ion concentration changes. J Clin Invest 1966;45:399-411.

5. Schreiber MD, Heymann MA, Soifer SJ. Increased arterial $\mathrm{pH}$, not decreased $\mathrm{PaCO}_{2}$ attenuates hypoxia-induced pulmo- 
nary vasoconstriction in newborn lambs. Pediatr Res 1986; 20:113-7.

6. Fullerton DA, Kirson LE, St. Cyr JA, Kinnard T, Whitman GJR. Influence of hydrogen ion concentration versus carbon dioxide tension on pulmonary vascular resistance after cardiac operation. J Thorac Cardiovasc Surg 1993; 106:528-36.

7. Morray JP, Lynn AM, Mansfield PB. Effect of $\mathrm{pH}$ and $\mathrm{PCO}_{2}$ on pulmonary and systemic hemodynamics after surgery in children with congenital heart disease and pulmonary hypertension. J Pediatr 1988;113:474-9.

8. Viitanen A, Salmenperä M, Heinonen J, Hynynen M. Pulmonary vascular resistance before and after cardiopulmonary bypass: the effect of $\mathrm{PaCO}_{2}$. Chest 1989;95:773-8.

9. Malik $\mathrm{AB}$, Kidd BSL. Independent effects of changes in $\mathrm{H}^{+}$ and $\mathrm{CO}_{2}$ concentrations on hypoxic pulmonary vasoconstriction. J Appl Physiol 1973;34:318-23.

10. Iannettoni MD, Bove EL, Mosca RS, Lupinetti FM, Dorostkar PC, Ludomirsky A, et al. Improving results with firststage palliation for hypoplastic left heart syndrome. J Thorac Cardiovasc Surg 1994;107:934-40.
11. Rossi AF, Sommer RJ, Lotvin A, et al. Usefulness of intermittent monitoring of mixed venous oxygen saturation after stage I palliation for hypoplastic left heart syndrome. Am J Cardiol 1994;73:1118-23.

12. Gullquist SD, Schmitz ML, Hannon GD, et al. Carbon dioxide in the inspired gas improves early postoperative survival in neonates with congenital heart disease following stage I palliation (Norwood). Circulation 1992;86(Suppl): I360.

13. Mora GA, Pizarro C, Jacobs ML, Norwood WI. Experimental model of single ventricle: influence of carbon dioxide on pulmonary vascular dynamics. Circulation 1994;90[Part 2]:II43-6.

14. Barnea O, Austin EH, Richman B, Santamore WP. Balancing the circulation: theoretic optimization of pulmonary/ systemic flow ratio in hypoplastic left heart syndrome. J Am Coll Cardiol 1994;24:1376-81.

15. Wessel DL, Adatia I, Giglia TM, Thompson JE, Kulik TJ. Use of inhaled nitric oxide and acetylcholine in the evaluation of pulmonary hypertension and endothelial function after cardiopulmonary bypass. Circulation 1993;88:2128-38. 